\title{
Microbe-derived acetate suppresses NAFLD/NASH development via hepatic FFAR2 signalling in mice
}

Ryo Aoki

Masayoshi Onuki

Koya Hattori

Masato Ito

Takahiro Yamada

Kohei Kamikado

Yun-Gi Kim

Noburiro Nakamoto

Ikuo Kimura

Julie M. Clarke

Takanori Kanai

Koji Hase

\section{Video Byte}

Keywords: Microbiome, non-alcoholic steatohepatitis, NASH, non-alcoholic fatty liver disease, NAFLD, short-chain fatty acids, acetate, prebiotics, prebiotic, inulin, FFAR2, Blautia, Bacteroides, insulin resistance, metabolism, metabolic syndrome, mouse study, mice, insulin, liver, hepatic, gut microbiome, intestine, portal vein, diet

Posted Date: October 13th, 2021

DOl: https://doi.org/10.21203/rs.3.rs-967519/v1

License: (9) This work is licensed under a Creative Commons Attribution 4.0 International License. Read Full License 


\section{Abstract}

In the US and east Asia, non-alcoholic fatty liver disease (NAFLD) affects at least a quarter of the population. This disease is the manifestation of metabolic syndrome in the liver and can progress to nonalcoholic steatohepatitis (NASH). The gut microbiome is likely a contributing factor in NAFLD development and progression. Recently, researchers sought to identify the mechanisms that link the two together. They used a high-fat/fructose/cholesterol diet (HFC) to induce NALFD-like symptoms in mice and found that adding the prebiotic inulin to their diet ameliorated these symptoms. Inulin-fed mice had global changes to their microbiome, particularly elevated levels of the bacterial groups Bacteroides and Blautia. Inulin supplementation also increased their gut concentrations of short-chain fatty acids, like acetate. Further experiments found that species from Bacteroides and Blautia had a synergistic effect on acetate production. and that it was acetate, not other short-chain fatty acids like butyrate or propionate, that protected against NAFLD/NASH. Reducing the expression of the acetate receptor FFAR2 reduced the protective effect of inulin and may increase insulin resistance in the liver. These results suggest that there is a commensal microbiome-acetate-FFAR2 molecular circuit that improves liver insulin sensitivity and prevents the development of NAFLD/NASH. 